\title{
LOCAL ANALYTIC FIRST INTEGRALS OF PLANAR ANALYTIC DIFFERENTIAL SYSTEMS
}

\author{
ILKER E. COLAK ${ }^{1}$, JAUME LLIBRE $^{1}$, AND CLAUDIA VALLS $^{2}$
}

\begin{abstract}
We study the existence of local analytic first integrals of a class of analytic differential systems in the plane, obtained from the Chua's system studied in $[6,7,11]$. The method used can be applied to other analytic differential systems.
\end{abstract}

\section{Introduction and Statement of Results}

The nonlinear ordinary differential equations appear in many branches of applied mathematics, physics and, in general, in applied sciences. For a differential system defined on the plane $\mathbb{R}^{2}$ the existence of a first integral determines completely its phase portrait. Since for such vector fields the notion of integrability is based on the existence of a first integral the following natural question arises: Given a differential system in $\mathbb{R}^{2}$, how to recognize if this system has a first integral?

The easiest planar differential systems having a first integral are the Hamiltonian ones. The integrable planar differential systems which are not Hamiltonian are, in general, very difficult to detect. Many different methods have been used for studying the existence of first integrals for non-Hamiltonian differential systems based on: Noether symmetries [4], the Darbouxian theory of integrability [8], the Lie symmetries [15], the Painlevé analysis [3], the use of Lax pairs [12], the direct method [9] and [10], the linear compatibility analysis method [17], the Carlemann embedding procedure [5] and [2], the quasimonomial formalism [3], the Ziglin's method [18], the Morales-Ramis theory [14], etc.

The objective of this paper is to show how to study the existence or nonexistence of analytic first integrals of planar analytic differential systems, when the standard theorems providing sufficient conditions for the nonexistence do not work.

We consider analytic differential systems

$$
\dot{x}=f(x, y), \quad \dot{y}=g(x, y),
$$

defined in an open subset $U$ of $\mathbb{R}^{2}$. We say that a non-constant analytic function $H: U \rightarrow \mathbb{R}^{2}$ is an analytic first integral of system (1) in $U$ if $H$ is constant on the solution curves of system (1), or equivalently

$$
f(x, y) H_{x}+g(x, y) H_{y}=0,
$$

in $U$. Of course, $H_{x}$ denotes the derivative of $H$ with respect to $x$.

2010 Mathematics Subject Classification. Primary: 34C05, 34A34, 34C14.

Key words and phrases. analytic first integral, analytic differential system. 
There exist well-known results providing sufficient conditions for the nonexistence of local analytic first integrals of system (1), as for instance the following two theorems.

Theorem 1 (Poincaré [16]). Assume that the eigenvalues $\lambda_{1} \neq 0$ and $\lambda_{2} \neq 0$ at some singular point $p$ of the analytic differential system (1) do not satisfy any resonance condition of the form

$$
\lambda_{1} k_{1}+\lambda_{2} k_{2}=0
$$

for any positive integers $k_{1}$ and $k_{2}$. Then system (1) has no analytic first integrals defined in a neighborhood of $p$.

Theorem 2 (Li, Llibre, Zhang [13]). Assume that the eigenvalues $\lambda_{1}$ and $\lambda_{2}$ at some singular point $p$ of the analytic differential system (1) satisfy that $\lambda_{1}=0$ and $\lambda_{2} \neq 0$. Then system (1) has no analytic first integrals in a neighborhood of $p$ if $p$ is isolated in the set of all singular points of system (1).

The problem for studying the non-existence of local analytic first integrals of system (1) in a neighborhood of a singular point appears when the sufficient conditions of Theorems 1 and 2 cannot be applied. In this work we deal with such a case. More precisely, we will study the local analytic integrability of the analytic differential system

$$
\begin{aligned}
& \dot{x}=y+m(\tanh x-x), \\
& \dot{y}=-(\alpha+1) y-\alpha m(\tanh x-x),
\end{aligned}
$$

in a neighborhood of its unique singular point, the origin. Here $\alpha$ and $m$ are real parameters. Our main result is the following.

Theorem 3. The analytic differential system (2) has a local analytic first integral in a neighborhood of the origin if and only if $m=0$. When $m=0$, a first integral is $(\alpha+1) x+y$.

Theorem 3 is proved in Section 2.

The differential analytic system (2) comes from the Chua's system

$$
\begin{aligned}
\dot{X} & =\alpha(Y-X)-\alpha\left(m_{1} X+\left(m_{0}-m_{1}\right) \tanh X\right), \\
\dot{Y} & =X-Y+Z, \\
\dot{Z} & =-(\beta Y+\gamma Z),
\end{aligned}
$$

studied in $[6,7,11]$, by doing the linear change of variables

$$
x=X, \quad y=\alpha(Y-X), \quad z=0,
$$

and by defining $m=\alpha m_{1}$, when $m_{0}=\beta=\gamma=0$.

\section{Proof of Theorem 3}

If $m=0$, it is easy to check that $(\alpha+1) x+y$ is an analytic first integral of system (2). 
Assume $m \neq 0$ and suppose $H$ is a local analytic first integral of system (2) in a neighborhood of the origin. Then we can write $H$ as a Taylor series

$$
H(x, y)=\sum_{i, j=0}^{\infty} a_{i, j} x^{i} y^{j},
$$

where $a_{0,0}=0$. We also expand the hyperbolic tangent in its Taylor series as

$\tanh x=x-\frac{x^{3}}{3}+\frac{2 x^{5}}{15}-\frac{17 x^{7}}{315}+\ldots=\sum_{i=1}^{\infty} \frac{B_{2 i} 4^{i}\left(4^{i}-1\right)}{(2 i) !} x^{2 i-1}=\sum_{i=1}^{\infty} b_{i} x^{2 i-1}$,

where the $B_{i}$ are the Bernoulli numbers, see for instance [1]. We note that the numbers $b_{i} \neq 0$ for $i \geq 1$. Then, clearly

$$
\tanh x-x=\sum_{i=2}^{\infty} b_{i} x^{i} y^{j} .
$$

By definition $H$ must satisfy the equation

$$
(y+m(\tanh x-x)) H_{x}+(-(1+\alpha) y-\alpha m(\tanh x-x)) H_{y}=0,
$$

which can be rewritten as

$$
G=m(\tanh x-x)\left(H_{x}-\alpha H_{y}\right)+y\left(H_{x}-(1+\alpha) H_{y}\right)=0,
$$

for all $(x, y)$.

Using equation (3) we will prove by induction that

$$
a_{i, n-i}=0 \quad \text { for all } n \geq 1 \text { and } i=1,2, \ldots, n,
$$

which means that all the $a_{i, j}$ are zero, hence $H$ is zero. We shall first compute the left hand side of equation (3). We have

$$
\begin{aligned}
H_{x} & =\sum_{i=1, j=0}^{\infty} i a_{i, j} x^{i-1} y^{j}=\sum_{i, j=0}^{\infty}(i+1) a_{i+1, j} x^{i} y^{j}, \\
H_{y} & =\sum_{i=0, j=1}^{\infty} j a_{i, j} x^{i} y^{j-1}=\sum_{i, j=0}^{\infty}(j+1) a_{i, j+1} x^{i} y^{j}, \\
H_{x}-\alpha H_{y} & =\sum_{i, j=0}^{\infty}\left((i+1) a_{i+1, j}-\alpha(j+1) a_{i, j+1}\right) x^{i} y^{j} \\
& =\sum_{i, j=0}^{\infty} c_{i, j} x^{i} y^{j}, \\
H_{x}-(\alpha+1) H_{y} & =\sum_{i, j=0}^{\infty}\left((i+1) a_{i+1, j}-(\alpha+1)(j+1) a_{i, j+1}\right) x^{i} y^{j} .
\end{aligned}
$$


Then

$$
\begin{aligned}
(\tanh x-x)\left(H_{x}-\alpha H_{y}\right)= & \sum_{j=0}^{\infty}\left(b_{2} c_{0, j} x^{3}+b_{2} c_{1, j} x^{4}+\left(b_{2} c_{2, j}+b_{3} c_{0, j}\right) x^{5}\right. \\
& \left.+\left(b_{2} c_{3, j}+b_{3} c_{1, j}\right) x^{6}+\ldots\right) y^{j} \\
= & \sum_{i=3, j=0}^{\infty}\left(\sum_{k=2}^{\lceil i / 2\rceil} b_{k} c_{i-2 k+1, j}\right) x^{i} y^{j}
\end{aligned}
$$

where $\lceil x\rceil$ denotes the ceiling function, which gives the smallest integer greater than $x$. We also have

$$
\begin{aligned}
y\left(H_{x}-(\alpha+1) H_{y}\right) & =\sum_{i, j=0}^{\infty}\left((i+1) a_{i+1, j}-(\alpha+1)(j+1) a_{i, j+1}\right) x^{i} y^{j+1} \\
& =\sum_{i=0, j=1}^{\infty}\left((i+1) a_{i+1, j-1}-(\alpha+1) j a_{i, j}\right) x^{i} y^{j}
\end{aligned}
$$

Using equalities (6) and (7), equation (3) becomes

$$
\begin{aligned}
G= & \sum_{i=0, j=1}^{\infty}\left((i+1) a_{i+1, j-1}-(\alpha+1) j a_{i, j}\right) x^{i} y^{j} \\
& +m \sum_{i=3, j=0}^{\infty}\left(\sum_{k=2}^{\lceil i / 2\rceil} b_{k} c_{i-2 k+1, j}\right) x^{i} y^{j}=0 .
\end{aligned}
$$

We first prove (4) for $n=1$. The coefficient of $y$ in $G$ gives

$$
a_{1,0}-(\alpha+1) a_{0,1}=0,
$$

whereas the coefficient of $x^{3}$ gives

$$
m b_{2} c_{0,0}=m b_{2}\left(a_{1,0}-\alpha a_{0,1}\right)=0 .
$$

Noting that neither $m$ nor $b_{2}$ are zero, equations (8) and (9) yield $a_{0,1}=$ $a_{1,0}=0$. So (4) is proved for $n=1$.

Now assume that (4) holds true for $k=1,2, \ldots, n$, for some arbitrary $n>1$, i.e. $a_{i, k-i}=0$ for $i=0,1, \ldots, k$, for each $k=1,2, \ldots, n$. We will show that $a_{i, n+1-i}=0$ for $i=0,1, \ldots, n+1$.

We say that the degree of the coefficient of a monomial is the degree of that monomial. Then each $a_{i, j}$, being the coefficients of the monomials in $H$, has degree $i+j$, where $i$ will be called its $x$-degree and $j$ will be called its $y$-degree. We remark that the coefficients $a_{i, j}$ of $H$ of odd degree and even degree are not interrelated. If we look at the coefficients of $\mathrm{G}$ of degree $n$, we see that

$$
\begin{aligned}
& \sum_{i=0}^{n}\left((i+1) a_{i+1, n-i-1}-(\alpha+1)(n-i) a_{i, n-i}\right) \\
& +m \sum_{i=3}^{n}\left(\sum_{k=2}^{\lceil i / 2\rceil} b_{k} c_{i-2 k+1, n-i}\right)=0,
\end{aligned}
$$


where

$$
c_{i-2 k+1, n-i}=(i-2 k+2) a_{i-2 k+2, n-i}-\alpha(n-i+1) a_{i-2 k+1, n-i+1} .
$$

The first series on the left hand side of equation (10) contains only the coefficients $a_{i, j}$ of $H$ of degree $n$. The second series, however, consists of coefficients of degree $n-2 k+2$, for $k=2, \ldots,\lceil n / 2\rceil$. Hence they are all of even degree if $n$ is even, and of odd degree if $n$ is odd.

In light of this remark, we will assume first that $n$ is odd.

First assume $\alpha \neq-1$. Looking at the coefficients of $G$ of degree $n+1$, we get

$$
\begin{aligned}
y^{n+1} & : a_{1, n}-(\alpha+1)(n+1) a_{0, n+1}=0, \\
x y^{n}: & 2 a_{2, n-1}-(\alpha+1) n a_{1, n}=0, \\
x^{2} y^{n-1}: & 3 a_{3, n-2}-(\alpha+1)(n-1) a_{2, n-1}=0, \\
x^{3} y^{n-2}: & 4 a_{4, n-3}-(\alpha+1)(n-2) a_{3, n-2}+m b_{2} c_{0, n-2}=0, \\
x^{4} y^{n-3}: & 5 a_{5, n-4}-(\alpha+1)(n-3) a_{4, n-3}+m b_{2} c_{1, n-3}=0, \\
x^{5} y^{n-4}: & 6 a_{6, n-5}-(\alpha+1)(n-4) a_{5, n-4}+m b_{2} c_{2, n-4}+m b_{3} c_{0, n-4}=0, \\
& \vdots \\
x^{n} y & :(n+1) a_{n+1,0}-(\alpha+1) a_{n, 1}+m b_{2} c_{n-3,1}+m b_{3} c_{n-5,1} \\
& +\ldots+m b_{\frac{n+1}{2}} c_{0,1}=0, \\
x^{n+1} & : m b_{2} c_{n-2,0}+m b_{3} c_{n-4,0}+\ldots+m b_{\frac{n+1}{2}} c_{1,0}=0 .
\end{aligned}
$$

For all the $c_{i, j}$ appearing in the above $n+2$ equations, $i+j \leq-2$. Then, by (5), they only contain $a_{i, j}$ with degree $i+j \leq n-1$, which are zero by the induction hypothesis. Hence all the $c_{i, j}$ above are zero. Therefore, the first $n+1$ equations recursively yield that all the $a_{i, n+1-i}$ are constant multiples of $a_{0, n+1}$. So, we only need to show $a_{0, n+1}=0$. The last equation holds trivially and contains no information.

Now we look at the coefficient of $x^{n+3}$ in $G$, which gives

$$
m b_{2} c_{n, 0}+m b_{3} c_{n-2,0}+\ldots+m b_{\frac{n+3}{2}} c_{1,0}=0 .
$$

Then, by the induction hypothesis and since $m b_{2} \neq 0$, equations (11) and (12) become

$$
(n+1) a_{n+1,0}-(\alpha+1) a_{n, 1}=0 \quad \text { and } \quad c_{n, 0}=(n+1) a_{n+1,0}-\alpha a_{n, 1}=0 \text {, }
$$

which yield that $a_{n, 1}=0$, and consequently $a_{0, n+1}=0$. This proves (4) for $n$ odd when $\alpha \neq-1$.

Now assume $\alpha=-1$. Then system (2) becomes

$$
\begin{aligned}
& \dot{x}=y+m(\tanh x-x), \\
& \dot{y}=m(\tanh x-x),
\end{aligned}
$$

and $G$ reduces to

$$
\sum_{i=0, j=1}^{\infty}(i+1) a_{i+1, j-1} x^{i} y^{j}+m \sum_{i=3, j=0}^{\infty}\left(\sum_{k=2}^{\lceil i / 2\rceil} b_{k} c_{i-2 k+1, j}\right) x^{i} y^{j}
$$


where

$$
c_{i, j}=(i+1) a_{i+1, j}+(j+1) a_{i, j+1} .
$$

We note as a special case that the coefficients of the monomials $y^{k+1}$, $x y^{k+1}$ and $x^{2} y^{k+1}$ of $G$ are, respectively, $a_{1, k}, 2 a_{2, k}$ and $3 a_{3, k}$, for all $k$. Then $G=0$ yields $a_{1, k}=a_{2, k}=a_{3, k}=0$ for all $k$.

We first look at the coefficients of $G$ of degree $n+1$ and we obtain

$$
\begin{aligned}
y^{n+1} & : a_{1, n}=0, \\
x y^{n}: & 2 a_{2, n-1}=0, \\
x^{2} y^{n-1}: & 3 a_{3, n-2}=0, \\
x^{3} y^{n-2}: & 4 a_{4, n-3}+m b_{2} c_{0, n-2}=0, \\
x^{4} y^{n-3}: & 5 a_{5, n-4}+m b_{2} c_{1, n-3}=0, \\
x^{5} y^{n-4}: & 6 a_{6, n-5}+m b_{2} c_{2, n-4}+m b_{3} c_{0, n-4}=0, \\
\vdots & :(n+1) a_{n+1,0}+m b_{2} c_{n-3,1}+\ldots+m b_{\frac{n+1}{2}} c_{0,1}=0, \\
x^{n} y & : \ldots+m b_{\frac{n+1}{2}} c_{1,0}=0 .
\end{aligned}
$$

Again, all the $c_{i, j}$ which appear above only contain $a_{i, j}$ with $i+j \leq n-1$, and hence are zero by the induction hypothesis. Then, the first $n+1$ of the above $n+2$ equations yield to

$$
a_{i, n+1-i}=0 \text { for } i=1, \ldots, n+1 .
$$

The last equation holds trivially. So it only remains to show that $a_{0, n+1}=0$.

Since $a_{0, n+1}$ has even degree, and the coefficients $a_{i, j}$ having even degree are not interrelated with those having odd degree, we next look at the coefficients of $G$ of degree $n+3$. We are going to write the $c_{i, j}$ explicitly, because not all of them will be zero as in the case of degree $n+1$. Thus we have

$$
\begin{aligned}
y^{n+3}: & a_{1, n+2}=0, \\
x y^{n+2}: & 2 a_{2, n+1}=0, \\
x^{2} y^{n+1}: & 3 a_{3, n}=0, \\
x^{3} y^{n}: & 4 a_{4, n-1}+m b_{2}\left(a_{1, n}+(n+1) a_{0, n+1}\right)=0, \\
x^{4} y^{n-1}: & 5 a_{5, n-2}+m b_{2}\left(2 a_{2, n-1}+n a_{1, n}\right)=0, \\
x^{5} y^{n-2}: & 6 a_{6, n-3}+m b_{2}\left(3 a_{3, n-2}+(n-1) a_{2, n-1}\right) \\
& \quad+m b_{3}\left(a_{1, n-2}+(n-1) a_{0, n-1}\right)=0, \\
\vdots & \\
x^{n+3}: & m b_{2}\left((n+1) a_{n+1,0}+a_{n, 1}\right)+\ldots+m b_{\frac{n+3}{2}}\left(2 a_{2,0}+a_{1,1}\right)=0
\end{aligned}
$$

The coefficient of $x^{n+3}$ consists of $a_{i, j}$ with degree $i+j \leq n+1$, whereas all the other coefficients of $G$ of degree $n+3$ contain a new unknown $a_{i, j}$ of degree $n+3$, see (13). Hence, equation (17) is where one expects to solve for $a_{0, n+1}$. But the coefficient of $x^{n+3}$ does not contain $a_{0, n+1}$. Also, it is zero due to (14) and the induction hypothesis. As a result, equation (17) 
does not give any information on $a_{0, n+1}$. However, we will see in a moment that all the necessary information about $a_{0, n+1}$ can be passed on to higher degree $a_{i, j}$.

By (14), $a_{1, n}=0$ in equation (16). Hence we have

$$
a_{4, n-1}=-\frac{(n+1) m b_{2}}{4} a_{0, n+1} .
$$

Moreover, using (14) and the induction hypothesis, from the coefficients of $x^{i} y^{n+3-i}$, for $i=4, \ldots, n+2$, we obtain

$$
a_{i, n+3-i}=0 \text { for } i=5, \ldots, n+3 .
$$

By (18), $a_{4, n-1}$ is a constant multiple of $a_{0, n+1}$. Then, showing $a_{0, n+1}=0$ is equivalent to showing $a_{4, n-1}=0$. So, we are going to look for more information about $a_{4, n-1}$ in the next set of equations obtained from the coefficients of $G$ of degree $n+5$.

Note that, for any $k$, the coefficient of $x^{k}$ in $G$ contains $a_{i, j}$ with $y$-degree $j=0,1$. So, in order to solve for $a_{0, n+1}$, we should pass the information about $a_{0, n+1}$ not only on to higher degrees, but also to lower $y$-degrees so that in the end we get a $y$-degree of 0 or 1 . When passing from $a_{0, n+1}$ to $a_{4, n-1}$, the goal of obtaining a lower $y$-degree is achieved.

The coefficients of $G$ of degree $n+5$ give

$$
\begin{aligned}
y^{n+5}: & a_{1, n+4}=0 \\
x y^{n+4}: & 2 a_{2, n+3}=0 \\
x^{2} y^{n+3}: & 3 a_{3, n+2}=0 \\
x^{3} y^{n+2}: & 4 a_{4, n+1}+m b_{2}\left(a_{1, n+2}+(n+3) a_{0, n+3}\right)=0 \\
x^{4} y^{n+1}: & 5 a_{5, n}+m b_{2}\left(2 a_{2, n+1}+(n+2) a_{1, n+2}\right)=0 \\
x^{5} y^{n}: & 6 a_{6, n-1}+m b_{2}\left(3 a_{3, n}+(n+1) a_{2, n+1}\right) \\
& +m b_{3}\left(a_{1, n}+(n+1) a_{0, n+1}\right)=0 \\
x^{6} y^{n-1}: & 7 a_{7, n-2}+m b_{2}\left(4 a_{4, n-1}+n a_{3, n}\right) \\
& +m b_{3}\left(2 a_{2, n-1}+n a_{1, n}\right)=0, \\
x^{7} y^{n-2}: & 8 a_{8, n-3}+m b_{2}\left(5 a_{5, n-2}+(n-1) a_{4, n-1}\right) \\
& +m b_{3}\left(3 a_{3, n-2}+(n-1) a_{2, n-1}\right) \\
& +m b_{4}\left(a_{1, n-2}+(n-1) a_{0, n-1}\right)=0, \\
x^{8} y^{n-3}: & 9 a_{9, n-4}+m b_{2}\left(6 a_{6, n-3}+(n-2) a_{5, n-2}\right) \\
& +m b_{3}\left(4 a_{4, n-3}+(n-2) a_{3, n-2}\right) \\
& +m b_{4}\left(2 a_{2, n-3}+(n-2) a_{1, n-2}\right)=0, \\
\vdots & \\
x^{n+5}: & m b_{2}\left((n+3) a_{n+3,0}+a_{n+2,1}\right)+\ldots+m b_{\frac{n+5}{2}}\left(2 a_{2,0}+a_{1,1}\right)=0 .
\end{aligned}
$$

By (14), (19) and the induction hypothesis, the coefficient of $x^{n+5}$ is also zero. This means that we cannot solve for $a_{4, n-1}$ either. But equations (20) 
and (21) contain the coefficient $a_{4, n-1}$. By (14) and (15), $a_{3, n}=a_{2, n-1}=$ $a_{1, n}=0$ in equation (20). Then we have

$$
a_{7, n-2}=-\frac{4 m b_{2}}{7} a_{4, n-1}
$$

Similarly by (14), (19) and the induction hypothesis, $a_{5, n-2}=a_{3, n-2}=$ $a_{2, n-1}=a_{1, n-2}=a_{0, n-1}=0$ in equation (21). Thus we get

$$
a_{8, n-3}=-\frac{(n-1) m b_{2}}{8} a_{4, n-1} .
$$

In addition, from the coefficients of $x^{i} y^{n+5-i}$, for $i=8, \ldots, n+4$, we get

$$
a_{i, n+5-i}=0 \text { for } i=9, \ldots, n+5 .
$$

We remark that as a consequence of (22) and (23), $a_{7, n-2}$ and $a_{8, n-3}$ are also constant multiples of $a_{4, n-1}$, and hence of $a_{0, n+1}$, pointing to the fact that they have the same sign. So, all the information about $a_{0, n+1}$ is now passed on to $a_{7, n-2}$ and $a_{8, n-3}$, which have degree $n+5$. We also note that $a_{7, n-2}$ and $a_{8, n-3}$ have lower $y$-degrees than $a_{4, n-1}$.

Next, we look at the coefficients of $G$ of degree $n+7$. The coefficient of $x^{n+7}$ is

$$
m b_{2}\left((n+5) a_{n+5,0}+a_{n+4,1}\right)+\ldots+m b_{\frac{n+7}{2}}\left(2 a_{2,0}+a_{1,1}\right) .
$$

It contains neither $a_{7, n-2}$ nor $a_{8, n-3}$, and is already zero by (14), (19), (24) and the induction hypothesis. The ones that do contain $a_{7, n-2}$ and $a_{8, n-3}$ are

$$
\begin{aligned}
x^{9} y^{n-2}: & 10 a_{10, n-3}+m b_{2}\left(7 a_{7, n-2}+(n-1) a_{6, n-1}\right) \\
& +m b_{3}\left(5 a_{5, n-2}+(n-1) a_{4, n-1}\right) \\
& +m b_{4}\left(3 a_{3, n-2}+(n-1) a_{2, n-1}\right) \\
& +m b_{5}\left(a_{1, n-2}+(n-1) a_{0, n-1}\right)=0, \\
x^{10} y^{n-3}: & 11 a_{11, n-4}+m b_{2}\left(8 a_{8, n-3}+(n-2) a_{7, n-2}\right) \\
& +m b_{3}\left(6 a_{6, n-3}+(n-2) a_{5, n-2}\right) \\
& +m b_{4}\left(4 a_{4, n-3}+(n-2) a_{3, n-2}\right) \\
& +m b_{5}\left(2 a_{2, n-3}+(n-2) a_{1, n-2}\right)=0, \\
x^{11} y^{n-4}: & 12 a_{12, n-5}+m b_{2}\left(9 a_{9, n-4}+(n-3) a_{8, n-3}\right) \\
& +m b_{3}\left(7 a_{7, n-4}+(n-3) a_{6, n-3}\right) \\
& +m b_{4}\left(5 a_{5, n-4}+(n-3) a_{4, n-3}\right) \\
& +m b_{5}\left(3 a_{3, n-4}+(n-3) a_{2, n-3}\right) \\
& +m b_{6}\left(a_{1, n-4}+(n-3) a_{0, n-3}\right)=0 .
\end{aligned}
$$

Among these three equations, (25) contains $a_{6, n-1}$, which has degree $n+5$ but cannot be determined by (24). So, we look at the other two equations.

By (14), (19), (24) and the induction hypothesis, equations (26) and (27) reduce to

$$
\begin{array}{r}
11 a_{11, n-4}+m b_{2}\left(8 a_{8, n-3}+(n-2) a_{7, n-2}\right)=0 \\
12 a_{12, n-5}+m b_{2}(n-3) a_{8, n-3}=0
\end{array}
$$


Then by (22) and (23), we deduce that $a_{11, n-4}$ and $a_{12, n-5}$ are also constant multiples of $a_{4, n-1}$, hence of $a_{0, n+1}$. Note that they have the same sign, and also lower $y$-degrees.

Furthermore, the coefficients of $x^{i} y^{n+7-i}$, for $i=12, \ldots, n+6$, write

$$
\begin{aligned}
x^{12} y^{n-5}: & 13 a_{13, n-6}+m b_{2}\left(10 a_{10, n-5}+(n-4) a_{9, n-4}\right) \\
& +m b_{3}\left(8 a_{8, n-5}+(n-4) a_{7, n-4}\right) \\
& +m b_{4}\left(6 a_{6, n-5}+(n-4) a_{5, n-4}\right) \\
& +m b_{5}\left(4 a_{4, n-5}+(n-4) a_{3, n-4}\right) \\
& +m b_{6}\left(2 a_{2, n-5}+(n-4) a_{1, n-4}\right)=0, \\
& \vdots \\
x^{n+6} y: & (n+7) a_{n+7,0}+m b_{2}\left((n+4) a_{n+4,1}+2 a_{n+3,2}\right) \\
& +\ldots+m b_{\frac{n+7}{2}}\left(a_{1,1}+2 a_{0,2}\right)=0 .
\end{aligned}
$$

By (14), (19), (24), and the induction hypothesis, the above $n-5$ equations yield

$$
a_{i, n+7-i}=0 \text { for } i=13, \ldots, n+7 .
$$

Observe that, in general, the coefficients of $G$ of degree $n+1+2 i$ recursively pass the information about $a_{0, n+1}$ on to $a_{i, j}$ with $y$-degrees as low as $n+1-2 i$. So, in order to pass the information about $a_{0, n+1}$ on to higher order $a_{i, j}$ with $y$-degrees 0 or 1 , we need to compute the coefficients of $G$ until degree $2 n+2$.

In light of this observation, if we continue computing the coefficients of $G$ up to degree $2 n+2$, we see that for $i=2, \ldots,(n+1) / 2$, among the coefficients of $G$ of degree $n+1+2 i$, the coefficient of $x^{4 i-2} y^{n-2 i+3}$ yields

$$
a_{4 i-1, n-2 i+2} \text { is a constant multiple of } a_{0, n+1} \text {, }
$$

and the coefficient of $x^{4 i-1} y^{n-2 i+2}$ gives

$$
a_{4 i, n-2 i+1} \text { is a constant multiple of } a_{0, n+1} \text {. }
$$

Moreover, $a_{4 i-1, n-2 i+2}$ and $a_{4 i, n-2 i+1}$ have the same sign. In addition, the coefficients of $x^{4 i+j} y^{n-2 i+1-j}$, for $j=0,1, \ldots, n-2 i$, show that

$$
a_{4 i+j, n-2 i+1-j}=0 \text { for } j=1,2, \ldots, n-2 i+1 .
$$

Finally, we look at the coefficient of $x^{2 n+4}$ and get

$$
\begin{aligned}
& m b_{2}\left((2 n+2) a_{2 n+2,0}+a_{2 n+1,1}\right)+m b_{3}\left(2 n a_{2 n, 0}+a_{2 n-1,1}\right) \\
& +m b_{4}\left((2 n-2) a_{2 n-2,0}+a_{2 n-3,1}\right)+\ldots+m b_{n+2}\left(2 a_{2,0}+a_{1,1}\right)=0 .
\end{aligned}
$$

In equation (31), the $a_{i, j}$ with $i+j \leq 2 n$ are zero by (30). Then equation (31) becomes

$$
(2 n+2) a_{2 n+2,0}+a_{2 n+1,1}=0,
$$

since $m b_{2} \neq 0$. By (28) and (29), $a_{2 n+1,1}$ and $a_{2 n+2,0}$ are constant multiples of $a_{0, n+1}$, and they have the same sign. Hence we conclude that $a_{0, n+1}=0$. This completes the proof of the theorem in the case $n$ odd.

The case when $n$ is even is proved in exactly the same way when $\alpha \neq-1$. When $\alpha=-1$, we need to compute the coefficients of $G$ until degree $2 n+3$ instead of $2 n+4$ when $n$ was odd. 
This proves (4), and the proof of Theorem 3 is established.

\section{ACKNOWLEDGMENTS}

The first author has been supported by AGAUR FI-DGR 2010. The second author has been supported by the grants MICINN/FEDER MTM 200903437, AGAUR 2009SGR 410 and ICREA Academia. The third author has been supported by AGAUR PIV-DGR-2010 and FCT through CAMGSD.

\section{REFERENCES}

[1] M. Abramowitz And I.A. Stegun, Handbook of mathematical functions with formulas, graphs, and mathematical tables, National Bureau of Standards Applied Mathematics Series, 55, Washington, D.C., 1964.

[2] R.F.S. Andrade And A. RAuH, The Lorenz model and the method of Carleman embedding, Phys. Lett. A 82 (1981), 276-278.

[3] T.C. Bountis, A. Ramani, B. Grammaticos And B. Dorizzi, On the complete and partial integrability of non-Hamiltonian systems, Phys. A 128 (1984), 268-288.

[4] F. Cantrijn And W. Sarlet, Generalizations of Noether's theorem in classical mechanics, SIAM Rev. 23 (1981), 467-494.

[5] T. Carleman, Application de la théorie des équations intégrales linéaires aux systémes d'équations différentielles non linéaires, Acta Mathematica 59 (1932), 6387.

[6] L. O. ChuA, A zoo of strange attractors from the canonical Chua's circuits, Proceedings of the IEEE 35th Midwest Symposium on Circuits and Systems, vol. 2, 1992, pp. 916-926.

[7] L. O. ChuA, A glimpse of nonlinear phenomena from Chua's oscillator, Philos. Trans. Roy. Soc. London Ser. A 353 (1995), no. 1701, 3-12.

[8] G. Darboux, Mémoire sur les équations différentielles algébriques du premier ordre et du premier degré (Mélanges), Bull. Sci. math. 2ème série 2 (1878), 60-96; 123-144; 151-200.

[9] H.J. Giacomini, C.E. Repetto and O.P. Zandron, Integrals of motion of threedimensional non-Hamiltonian dynamical systems, J. Phys. A 24 (1991), 4567-4574.

[10] J. Hietarinta, Direct methods for the search of the second invariant, Phys. Rep. 147 (1987), 87-154.

[11] N. V. Kuznetsov, G. A. Leonov and V. I. Vagaitsev, Hidden attractors in smooth Chua's systems, preprint.

[12] P.D. LAx, Integrals of Nonlinear Equations of Evolution and Solitary Waves, Commun. Pure Appl. Math. 21, (1968), 467-490.

[13] W. Li, J. Llibre AND X. Zhang, Local first integrals of differential systems and diffeomorphisms, Z. Angew. Math. Phys. 54 (2003), 1-21.

[14] J.J. Morales-Ruiz, Differential Galois Theory and non-integrability of Hamiltonian systems, Progress in Math. Vol. 178, Birkhauser, Verlag, Basel, 1999.

[15] P.J. OLver, Applications of Lie groups to differential equations, Springer, New York, 1986.

[16] H. Poincaré, Mémoire sur les courbes définies par les équations différentielles (Oeuvreus de Henri Poincaré, I), Gauthiers-Villars, Paris, 1951, pp. 95-114.

[17] J.M. Strelcyn and S. Wojciechowski, A method of finding integrals for threedimensional dynamical systems, Phys. Lett. A 133 (1988), 207-212.

[18] S. Ziglin, Branching of Solutions and the nonexistence of first integrals in Hamiltonian Mechanics. II, Funct. Anal. and Its Appl. 17 (1983), 6-17. 
${ }^{1}$ Departament de Matemàtiques, Universitat Autònoma de Barcelona, 08193 Bellaterra, Barcelona, Catalonia, Spain

E-mail address: ilkercolak@mat.uab.cat, jllibre@mat.uab.cat

${ }^{2}$ Departamento de Matemática, Instituto Superior Técnico, Universidade TÉCnica de Lisboa, 1049-001 Lisboa, Portugal

E-mail address: cvalls@math.ist.utl.pt 